\title{
Molecular pathogenesis of endometriosis-associated clear cell carcinoma of the ovary (Review)
}

\author{
HIROSHI KOBAYASHI, HIROTAKA KAJIWARA, SEIJI KANAYAMA, YOSHIHIKO YAMADA, \\ NAOTO FURUKAWA, TAKETOSHI NOGUCHI, SHOJI HARUTA, SHOZO YOSHIDA, \\ MARIKO SAKATA, TOSHIYUKI SADO and HIDEKAZU OI
}

Department of Obstetrics and Gynecology, Nara Medical University, 840 Shijo-cho, Kashihara 634-8522, Japan

Received February 10, 2009; Accepted April 29, 2009

DOI: $10.3892 /$ or_00000429

\begin{abstract}
Epithelial ovarian cancer (EOC) is the leading cause of death in women with gynecological malignancies. Among EOC, clear cell carcinoma (CCC) and endometrioid adenocarcinoma (EAC) differ from the other histological types with respect to their clinical characteristics and carcinogenesis. Both tumor types are often associated with endometriosis. EAC is recently reported to be characterized by K-RAS activation and PTEN dysfunction. However, the molecular changes in $\mathrm{CCC}$ remain largely unknown. The aim of this review is to summarize the current knowledge on the molecular mechanisms involved in CCC tumorigenesis. The present article reviews the English language literature for biological, pathogenetic and pathophysiological studies on endometriosis-associated CCC of the ovary. Several recent studies of loss of heterozygosity (LOH), allelic loss, comparative genomic hybridization, mutation, methylation status, microarray gene-expression profiling and proteomics are discussed in the context of CCC biology. Retrograde menstruation or ovarian hemorrhage carries highly prooxidant factors, such as heme and iron, into the peritoneal cavity or ovarian endometrioma. A histologically normal ectopic endometrium bears genetic damages caused by irondependent oxidative stress. DNA damage or LOH caused by oxidative stress is a critical factor in the carcinogenic process. LOH studies have implicated the involvement of specific chromosomal regions $(5 \mathrm{q}, 6 \mathrm{q}, 9 \mathrm{p}, 10 \mathrm{q}, 11 \mathrm{q}, 17 \mathrm{q}$ and 22q). Furthermore, the PTEN and APC (early event), p53, polo-like kinases, Emi1 and K-RAS (late event) genes may be involved in CCC carcinogenesis. The molecular pathology of CCC is heterogeneous and involves various putative precursor lesions and multiple pathways of development, possibly via genetic alteration by oxidative stress.
\end{abstract}

Correspondence to: Dr Hiroshi Kobayashi, Department of Obstetrics and Gynecology, Nara Medical University, 840 Shijocho, Kashihara 634-8522, Japan

E-mail: hirokoba@naramed-u.ac.jp

Key words: endometriosis, clear cell carcinoma, oxidative stress, loss of heterozygosity

\section{Contents}

1. Introduction

2. Identification of relevant studies

3. Characteristics of CCC

4. $\mathrm{LOH}$ analysis

5. LOH involved in endometrioid adenocarcinoma (EAC) of the ovary

6. LOH involved in clear cell carcinoma (CCC) of the ovary: the candidate tumor suppressor genes

7. Microsatellite instability in CCC

8. Genes specifically up-regulated in $\mathrm{CCC}$

9. Clear cell adenofibroma-CCC sequence

10. Iron, oxidative stress and carcinogenesis

11. Conclusions

\section{Introduction}

Epithelial ovarian cancer (EOC) is the leading cause of death in women with gynecological malignancies worldwide. The majority of patients present with stage III and IV disease, for which the 5-year survival rates are less than $20 \%$. Most of the patients with EOC present with advanced disease that is not cured by surgery. Ninety per cent of EOC are derived from epithelium within inclusion cysts or from the ovarian surface epithelium and these neoplasms are classified into serous, mucinous, endometrioid adenocarcinoma (EAC), clear cell carcinoma (CCC) and other types (1).

Molecular genetic alterations play a key role in carcinogenesis (1). High-grade serous carcinoma arises in a de novo fashion and is characterized by p53 mutations and BRCA1 and/or BRCA2 dysfunction. In contrast, low-grade serous carcinomas are characterized by activation of the RAS-RAF signaling pathway secondary to mutations in KRAS and BRAF in an adenoma-borderline tumor-carcinoma sequence. Similarly, mucinous carcinomas have also K-RAS mutations and probably develop via an adenoma-carcinoma sequence. K-RAS mutation is considered to be an early event in mucinous tumorigenesis.

Substantial histopathology data provide evidence that endo-metriosis might be viewed as a neoplastic process of CCC and EAC tumors, possibly via intermediary atypical borderline lesions. Endometriosis is common, with an estimated incidence of $10-15 \%$ of women in their reproductive 
age. The implantation and the metaplasia theories describe the mechanism of initiation of endometriotic lesions. Epidemiology studies have demonstrated a strong link between endometriosis and the CCC and EAC subtypes (2). Recent study in Japan also showed that endometriosisassociated ovarian carcinoma (EAOC) is not rare, with an estimated incidence of $0.72 \%$ (3). Thus, atypical endometriosis is considered to be the precursor lesion of both tumor types (2). In addition, the recent studies showed that clear cell adenofibroma (CCAF) may also be a clonal precursor lesion in CCC (4). Many of the risk factors (earlier menarche, more regular periods, shorter cycle lengths and lower parity) associated with endometriosis and EOC are the same. The genes involved in both endometriosis and EOC have been shown to play a role in the pathogenesis of EAOC.

EAOC is considered to arise in a stepwise fashion in an adenoma-carcinoma sequence from typical to micropapillary borderline tumors to invasive carcinoma. In a model of genetically engineered mice harboring an oncogenic allele of K-RAS resulting in benign lesions reminiscent of endometriosis, a deletion of PTEN caused progression towards the EAC, but not the CCC (5). K-RAS mutation has been detected in EAC tissue, but not in atypical endometriosis bordering the cancerous region (6). Low-grade EAC are strongly characterized by mutations in PTEN, the Wnt/CTNNB1 pathway, and microsatellite instability (MSI) (1). High-grade EAC have similar changes to high-grade serous carcinomas, possibly via p53 mutations and BRCA1/2 dysfunction. However, recent molecular genetic studies have shown significantly lower TP53 mutation rates in CCC than in other types of EOC. Thus, the molecular changes in CCC of the ovary remain largely unknown.

The aim of this review is to summarize the current knowledge of the molecular mechanisms involved in EAOC, especially CCC, including results of allelic loss, LOH, comparative genomic hybridization, mutation, methylation, microarray gene-expression profiling and proteomics studies. The identified molecular changes and pathways of development in CCC will facilitate the development of more sensitive means of diagnosis and tailored therapies for this malignancy, resulting in better clinical outcome.

\section{Identification of relevant studies}

The present article reviews the English language literature for biological, pathogenetic and pathophysiological studies on endometriosis-associated CCC of the ovary. We searched MEDLINE (PubMed) electronic databases for a 20-year period (1989-2008), combining the keywords 'loss of heterozygosity' 'allelic loss' 'comparative genomic hybridization' 'mutation' 'methylation' 'microarray' 'gene expression profiling' or 'proteomics' with 'clear cell carcinoma of the ovary' or 'endometriosis'. Several recent studies are discussed in the context of CCC biology. Additionally, references in each article were searched to identify potentially missed studies. Study selection is as follows. Case series of ovarian cancer that reported the histological characteristics of EOC were included. For studies that reported data on the nonserous subtypes, only data pertaining to CCC and EAC were included. Studies that only categorized histologic subtype as serous and non-serous, without further classification of nonserous subtypes were excluded. Here, we discuss possible carcinogenic pathways for endometriosis-associated CCC.

\section{Characteristics of CCC}

CCC of the ovary was initially termed as 'mesonephroma' (7). They account for $4-12 \%$ of all EOC worldwide. In contrast, the frequency of CCC ( $>20 \%$ of all EOC) has been shown to be very common in Japan. When compared with their serous counterparts, a greater proportion of CCC tumors present as early-stage tumors, up to $60 \%$ of the patients with CCC have stage I disease. They show resistance to chemotherapy and result in poor prognosis. An increased incidence of vascular thrombotic events is seen in this disease. Gene expression profiling studies have shown marked similarities with CCC of the kidney and endometrium (7) and ovary (8), suggesting that certain molecular events may be common to CCC regardless of the organ of origin (8). This raises interesting possibilities regarding novel therapeutic approaches.

\section{4. $\mathrm{LOH}$ analysis}

Activation of oncogenes, deregulation of tumor suppressor genes, up-regulation of growth factors/growth factor receptor signaling cascade systems, and alterations in apoptosis regulation, have been reported to play important roles in the process of carcinogenesis. Inactivation of tumor suppressor genes derives from allelic loss (homozygous deletion or $\mathrm{LOH})$, mutation and methylation of these genes. Aberrant hypermethylation is correlated with microsatellite instability (MSI). LOH analysis has been extensively used to study chromosomal loci that have been linked to cancer.

In early stage of endometriosis, the cells could begin with genomic abnormalities involving implantation, metaplasia and growth processes (9), followed by somatic mutations, activation of oncogenes, or inactivation of tumor suppressor genes with an identified 'second hit' (10). By the time these implants or metaplastic lesions have developed into small endometriotic cysts, about one-third will harbor $\mathrm{LOH}$ (9,11-13). LOH has commonly been identified in endometriosis at 1q, 5q, 6q, 9p, 10q, 11q, 17p, 22q (9,11-13). Comparative genomic hybridization $(\mathrm{CGH})$ repeated in endometriotic tissue revealed loss of DNA copy number on $1 p, 22 q$ and chromosome $X$ (14). This genetic alteration could be necessary for the survival of ectopic endometrial tissue, but other mutations or inactivation of tumor suppressor genes are required for the promotion of a premalignant state. Prowse et al (2) have reported that LOH in both the endometriosis and EOC was detected on chromosomes 3q, 4q, 5q, $6 \mathrm{p}, 6 \mathrm{q}, 7 \mathrm{q}, 10 \mathrm{q}, 11 \mathrm{p} \mathrm{11q,13q,14q,16q,17q}$ and $21 \mathrm{q}$. Additional LOH events were detected in the EOC alone on chromosomes 2p, 2q, 3p, 5p, 6q, 7p, 8q, 9p, 10p, 12q, 14p, 17 p, 18p, 18q, 19p, 20q and 22q.

\section{LOH involved in endometrioid adenocarcinoma (EAC) of the ovary}

LOH on the chromosome regions, including 4q, 5q13-q14, 6q14-q15, 9p21, 10p23.3, 11q23 and 22q13, has been shown 
to be very common in EAC (15). Mutations of PTEN (chromosome 10p23.3) were identified in $20 \%$ of both EAC and ovarian endometriosis, suggesting that the PTEN inactivation is an early event in the malignant process of endometriosis $(14,16)$. A separate study identified reduced PTEN protein expression in $15 \%$ of endometriosis cases (17). The second hit or carcinogenesis step could be represented by the reduced expression of hMLH1, MSI (17).

\section{LOH involved in clear cell carcinoma (CCC) of the ovary: the candidate tumor suppressor genes}

$\mathrm{LOH}$ events were common in $\mathrm{CCC}$ and synchronous endometriotic lesions $(15,17)$. $\mathrm{LOH}$ on the chromosome regions, including 5q, 6q, 9p, 11q, 17q and 22q, has been shown to be common in CCC (15). Although loss of p53 gene accompanies the transition to atypical endometriosis, mutations in p53 are rare or conspicuously absent in CCC (7). Similar to EAC, loss of PTEN expression has been noted in $40 \%$ of early-stage CCC, suggesting that PTEN inactivation may also be an early event in CCC development (7). LOH of PTEN exacerbates genetic instability.

Numerous studies have shown that $\mathrm{LOH}$ at the following chromosomal regions may be involved in the development of endometriosis-associated CCC. These loci may contain candidate tumor suppressor genes involved in the initiation, promotion or progression of CCC but results obtained are sometimes discordant and not established.

1p. $\mathrm{LOH}$ on $1 \mathrm{p}$ is rare in clear cell adenofibroma (CCAF) but frequent in CCC (4). The following tumor suppressor genes have been reported on chromosome 1p. TP73 (tumor protein $\mathrm{p} 73$, location at $1 \mathrm{p} 36.3$ ): this gene encodes tumor protein $\mathrm{p} 73$, which is a member of the p53 family of transcription factors involved in cellular responses to stress and development. DIRAS3 (DIRAS family, GTP-binding RASlike 3): location, $1 \mathrm{p} 31$. This gene is a member of the RAS superfamily. Its expression is associated with growth suppression of ovarian cancer. APITD1 (apoptosis-inducing, TAF9-like domain 1): location, 1p36.22. This was identified in the neuroblastoma tumor suppressor candidate gene.

$1 q$. The genes for galactose-1-phosphate uridyl transferase (GALT) and apolipoprotein A2 (APOA2) have been mapped to chromosome 1q (11). Microsatellite DNA assays reveal an allelic imbalance in GALT and APOA2 loci in patients with endometriosis (11). SELENBP1 (selenium binding protein 1): location, 1q21-q22. It has been proposed that the effects of selenium in preventing cancer and neurologic diseases may be mediated by selenium-binding proteins.

$3 p$. Multiple chromosome $3 p$ tumor suppressor genes have been proposed in the pathogenesis of EOC (18). A deletion involving chromosome $3 p$ frequently occurs in small cell lung cancer (19). There are many tumor suppressor genes located at chromosome 3p, including DRR1 (down-regulated in renal cell carcinoma 1), BAP1 (BRCA1-associated protein 1), ARP (arginine-rich, mutated in early stage tumors; ARMET), RIS1 (RAS-induced senescence 1), LIMD1 (LIM domains-containing one), and LTF (Lactotransferrin) (20).
$4 q$. The candidate tumor suppressors located within chromosome $4 \mathrm{q}$ in hepatocellular and ovarian carcinomas (15).

$5 q$. $\mathrm{LOH}$ on $5 \mathrm{q} 13-14$ was rare in endometriosis components but frequent in EAC components (15). APC (adenomatous polyposis coli): location, 5q21-q22. This gene encodes a famous tumor suppressor protein that acts as an antagonist of the Wnt signaling pathway. Defects in this gene cause familial adenomatous polyposis, an autosomal dominant premalignant disease that usually progresses to malignancy, including colon carcinoma. IRF1 (interferon regulatory factor-1): location, 5q31.1. IRF1 functions as a transcription activator of genes induced by interferons $\alpha, \beta$ and $\gamma$. This gene has been shown to play roles in regulating apoptosis and tumor-suppression.

$6 p . \mathrm{LOH}$ in both endometriosis and carcinoma was detected on chromosomes $6 \mathrm{p}(2)$.

$6 q$. The genes for estrogen receptor and superoxide dismutase, a tumor suppressor gene, have been mapped to chromosome 6q $(9,12,15)$. SNORD50A (small nucleolar RNA, C/D box 50A): location, 6q14.3. This gene functions as a reasonable candidate for the tumor suppressor gene in prostate cancer and likely in other types of cancers. PLAGL1 (pleiomorphic adenoma gene-like 1): location, 6q24-q25. This gene might be involved in regulating the $\mathrm{p} 21^{\mathrm{WAF} 1 / \mathrm{Cip} 1}$ gene and protein expression through its protein-protein interaction with p53 and histone deacetylase 1 (HDAC1).

9p. The CDKN2 (p16Ink4) gene, encoding the p16 protein, has been mapped to chromosome 9p21 $(9,11,15)$. p16 is one of the cycle-dependent kinase inhibitors and acts as a negative cell-cycle regulator (21). It is a potent tumor-suppressor gene (21). Inactivation of p16 gene may derive from LOH, homozygous deletion, mutation and methylation of this gene. Mutations and hypermethylation of the promoter region of p16 are rare events in EOC $(17,22)$. Chromosome $9 \mathrm{p}$ deletions have also been noted in CCC of the kidney (7). Furthermore, diffuse positivity with p16 in the uterine cervix can be regarded as a surrogate marker of the presence of high-risk human papillomavirus (HPV). EOC are usually p16positive and endometriosis is p16-negative or focally positive (23). p16 alteration may occur independently of histologic subtype of EOC (24). However, the p16 overexpression does not mean the suppression of tumor cell proliferation. In addition, alterations in the various cell cycle regulatory molecules or tumor suppressor genes, particularly of p21, one of the p16 family members, and cyclin E, might be related to the biological characteristics of CCC (24).

10q. Genetic aberrations, such as LOH and somatic mutations leading to functional inactivation of the PTEN tumor suppressor gene, located on chromosome 10q23.3, have been identified in endometriosis $(20 \%)(12,16)$ and one third of EOC (25). the frequency of LOH at 10q23.3 and PTEN somatic mutations in CCC and EAC was still higher than in other histological types of EOC, although LOH occurred less frequently in CCC $(8 \%)$ than in EAC $(20 \%)(16,26,27)$. PTEN gene alterations are very early events in the development of 
EAC and also support the concept that endometrial cysts are a precancerous form of EAC. Dinulescu et al (5) reported that, in an animal model, tissue-specific expression of active mutant K-RAS results in pelvic endometriosis, or tissuespecific expression of active mutant K-RAS and inactivation of PTEN results in metastatic EAC. There are two important reports that somatic mutations in the PTEN gene is an early event in the development of endometriosis-CCC sequence (16) and CCAF-CCC sequence (4). BRWD2 (bromodomain and WD repeat domain containing 2): location, 10q26. This gene is located in the chromosome 10q25-26 region, which is frequently deleted in gliomas and tumors of other tissues.

11q. Frequent allelic loss at chromosome 11q23-q24 occurs in a wide variety of human cancers, including breast and ovarian tumors, suggesting that this region may harbor a tumor suppressor gene $(9,15)$. BCSC-1 (breast cancer suppressor candidate-1) may exert a tumor suppressor activity and is a likely target of the LOH observed on 11q23-q24 in cancer (28). CADM1 (cell adhesion molecule 1): location, 11q23.2. Down-regulation of CADM1 tumor suppressor gene expression is a critical event in neuroblastoma pathogenesis resulting in tumor progression and unfavorable patient outcome. Progesterone receptor gene is also located on chromosome 11q23 $(9,15)$.

$13 q$. LOH on $13 q$ is rare in CCAF but frequent in CCC (2). RCBTB1 (regulator of chromosome condensation and BTB domain containing protein-1). This gene maps to a region of chromosome 13q14 that is frequently deleted in B-cell chronic lymphocytic leukemia and other lymphoid malignancies. Several observations of the coexistence of endometriosis and cancer have been published. Elevated risks were found for not only ovarian cancer but also lymphoma (29).

16q. Allelic imbalances affecting the long arm of chromosome 16 have been extensively reported in the literature as common abnormalities observed in various carcinoma types. WWOX that spans a fragile region of DNA located at 16q23.3-24.1 is a candidate tumor suppressor gene (30). The two histotypes showing significant loss of WWOX expression were of the CCC and mucinous types.

$17 p$. The $17 \mathrm{p} 13$ genetic locus is known to harbor the tumor suppressor gene p53 (11). The TP53 gene is the most frequently mutated gene in human cancers and plays a role as a transcription factor, causing cell cycle arrest or cell death. Germline mutations of TP53 have usually been observed to occur in the highly conserved region of exons 5-8. The majority of TP53 missense mutations occur in its DNAbinding domain. Mutations in the TP53 gene were observed in one-half of the EOC. Mutation of TP53 is an early event in the development of EOC and is present prior to stromal invasion. In serous and endometrioid carcinomas, staining for p53 was usually positive and diffuse (24). P53 mutation was more common in EAC than in CCC (27). TP53 alterations occur frequently in late stage endometriosis (10). atypical endometriosis had p53 overexpression (31). Thus, p53 mutations may affect malignant transformation of endometriosis into EAC (16) or CCC (32). HIC1 (hypermethylated in cancer-1): location, $17 \mathrm{p} 13.3$. This gene is another tumor suppressor gene. Inactivation of HIC1 by hypermethylation is associated with several cancer pathogenesis.

$17 q$. The histologies of EOC displaying a pattern of $17 q$ deletions were of the EAC, CCC and mucinous types (33). Tumor suppressor genes on $17 \mathrm{q}$ are possibly involved in development of endometriosis (2,34). NME1 (non-metastatic cells 1, protein (NM23A) expressed in): location, 17q21.3. This gene was identified because of its reduced mRNA transcript levels in highly metastatic tumors.

$22 q$. LOH at $22 q$ has been identified in some endometrial cysts and CCC $(9,156)$. There are some tumor suppressor genes on chromosome 22q. SMARCB1 (SWI/SNF related, matrix associated, actin dependent regulator of chromatin, subfamily b, member-1) is located on chromosome $22 \mathrm{q} 11$. Mutations in this gene have been associated with malignant rhabdoid tumors. NF2 neurofibromin 2 (merlin): location, $22 q 12.2$. Mutations in this gene are associated with neurofibromatosis type II.

\section{Microsatellite instability in CCC}

Microsatellite instability (MSI) is a phenomenon that is well characterized in mismatch repair (MMR)-deficient tumor cells. The MMR pathway is well defined in both inherited and sporadic cancer pathogenesis. For example, hyperplastic polyps in colon are part of the distinct pathway of colorectal carcinogenesis characterized by MSI and deficiency in DNA MMR. Although the relationship between endometriosis and MSI has been controversial (6), a high frequency ( $\sim 80 \%)$ of MSI was found in endometriosis and atypical endometriosis $(14,25)$. In addition, methylation phenotype of hMLH1 gene (whose gene product is a component of the DNA MMR pathway) is correlated with microsatellite phenotype of MMR genes. Hypermethylation of hMLH1 is noted in $\sim 10 \%$ of endometriosis (17). The high-level MSI or MSI leading to the PTEN dysfunction has also been reported for EOC with frequencies varying from 6 to $37 \%(14,25,35)$. Low-grade EAC are predominantly characterized by MSI. These data suggest that MSI is an early event in the development of EAOC.

MSI is seen in some chronically inflamed tissues even in the absence of genetic inactivation of the MMR system (36). Oxidative stress associated with chronic inflammation might damage protein components of the MMR system, leading to its functional inactivation. These data allow us to speculate that inactivation of the MMR function in response to oxidative stress may be responsible for the MSI seen in non-neoplastic (endometriosis) and cancer tissues (CCC) associated with chronic inflammation (see 'Iron, oxidative stress and carcinogenesis' section).

\section{Genes specifically up-regulated in CCC}

An enormous amount of knowledge has been obtained from recent preclinical experiments. Mutations of K-RAS and upregulated HNF-1ß, Emi1 and mTOR signaling are specifically found in $\mathrm{CCC}$. 


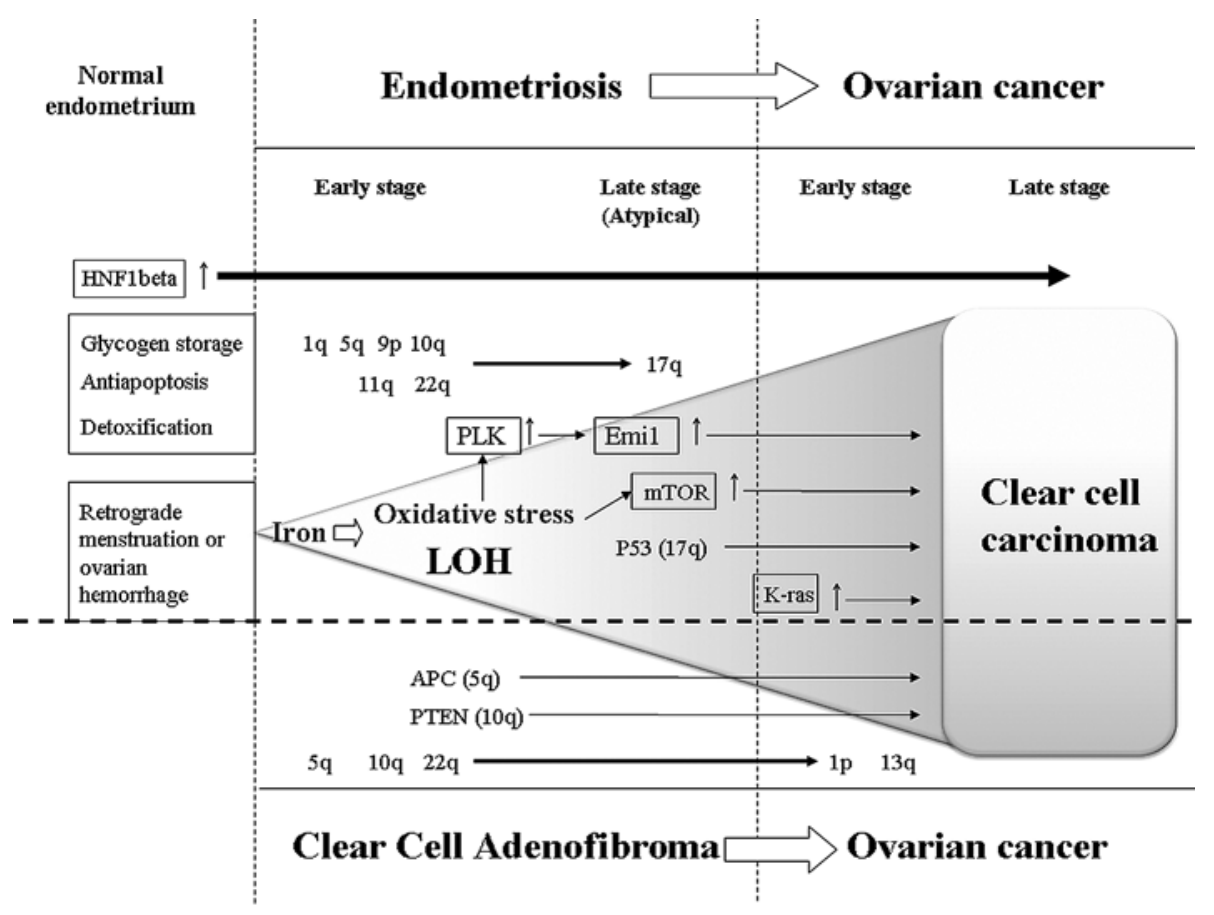

Figure 1. Proposed genetic alterations in the development of CCC. The genetic model of CCC carcinogenesis arising in endometriosis and CCAF is shown.

$K-R A S$. K-RAS mutations occur significantly more frequently in mucinous and EAC tumors, but not in normal or atypical endometriosis bordering the cancerous region (6). Therefore, $\mathrm{K}-\mathrm{RAS}$ mutations may be associated with the late event in the endometriosis-CCC sequence (37).

$H N F-1 \beta$. It has recently been reported that expression of HNF-1ß, a transcription factor, may be a genetic common lineage among the mid-to-late secretory endometrium, endometriosis, atypical endometriosis and CCC (38). Since CCC are characterized by overexpression of $\mathrm{HNF}-1 \beta$ gene and protein (38), HNF-1ß is a useful biomarker that could aid in the diagnosis of CCC (39). Hypomethylation of the HNF-1B $\mathrm{CpG}$ island participates in the HNF-1B up-regulation in CCC (40). A majority of biological functions of CCC is governed HNF-1ß-dependently and possibly by interacting pathways. HNF-1B was essential for its survival for CCC and possibly endometriosis and involved in the characteristics of CCC, including glycogen accumulation in cancer cells (Periodic acid-Schiff is positive for glycogen in the cytoplasm), strong antiapoptotic activity, and chemoresistance due to lower cell proliferation (41). Gene expression profiling studies have shown that several important CCC-specific genes overlapped with those known to be regulated by HNF-1ß (42). Thus, HNF-1B is not only an excellent CCC-specific molecular marker but also a molecular target for therapy of ovarian CCC (41).

Emil. Polo-like kinases (PLK) have been implicated in mitotic regulation and carcinogenesis. High expression levels of PLK in ectopic endometrial cells are associated with abnormal cell cycle regulation of endometriosis, suggesting that PLK may play important roles in the pathogenesis of endometriosis (45).
Interestingly, PLK is rapidly activated by reactive oxygen species or hydrogen peroxide and promotes genome stability by regulating DNA replication under stressful conditions. PLK phosphorylates Emil (Early mitotic inhibitor-1) to ensure mitosis entry. Emi1 is a key cell cycle regulator, that promotes $\mathrm{S}$-phase and mitotic entry by inhibiting the anaphase promoting complex/cyclosome (APC/C) (44). Emil overexpression causes mitotic catastrophe and genomic instability, and contributes to tumorigenesis (44). Recent study demonstrated that the Emi1/APC/C pathway is up-regulated and active in atypical endometriosis component during clear cell tumorigenesis (45). Subsequent steps in CCC carcinogenesis may involve the Emi1-dependent MSI, since overexpression of Emi1 leads to genomic instability.

mTOR. The mammalian target of rapamycin (mTOR) has been identified as a downstream target of the PI3K/Akt pathway. mTOR has emerged as a critical effector in cellsignaling pathways commonly deregulated in human cancers. We have previously reported that mTOR is phosphorylated and activated in endometriosis and CCC specimens (46). This leads to phosphorylation of downstream targets, p70S6K and 4E-BP1, and the subsequent enhanced translation of mRNAs that are critical for cell cycle progression and proliferation. mTOR signaling has emerged as an attractive therapeutic target for cancer therapy. The potential applications of mTOR inhibitors for treating various types of cancer have been actively studied both preclinically and clinically.

The Wnt and its downstream pathway ß-catenin is another key developmental pathway for which alterations have been described in various human cancers. Over-activation of the transcription factor $\beta$-catenin has also been associated with malignancy such as colon and breast cancer. Wnt/ß-catenin 
activation can stimulate the downstream protein translation via mTOR activation (47). The activity of B-catenin can be stimulated by mechanisms other than via the Wnt. Interestingly, $\beta$-catenin functions as a pivotal molecule in defense against oxidative stress.

\section{Clear cell adenofibroma-CCC sequence}

Most clear cell neoplasms of the ovary are carcinomas; benign and borderline clear cell tumors (adenofibromas; CCAF) are uncommon. Some reports suggest that CCC can arise from not only endometriosis but also clear cell adenofibromas (4). Like endometriosis-associated CCC, genetic alterations on chromosomes $5 \mathrm{q}, 10 \mathrm{q}$ and $22 \mathrm{q}$ in CCAF may be early events in carcinogenesis via the 'CCAF-CCC sequence' (4). The genetic instability and $\mathrm{LOH}$ may lead to possible alterations of known tumor suppressor genes, such as the APC (adenomatous polyposis coli) gene on chromosome 5q21-q22 and the PTEN gene on 10q23.3. LOH on 1p and 13q increased in accordance with higher cytologic atypia: much more frequent in CCC (4), suggesting that these alleles may be involved later in the $\mathrm{CCAF}-\mathrm{CCC}$ sequence.

\section{Iron, oxidative stress and carcinogenesis}

The investigators highlight some major achievements in the study of DNA damage caused by oxygen-free radicals and the role in carcinogenesis played by oxidatively damaged DNA (48). Heme and free iron are pro-oxidant and can induce oxidative stress and DNA damage. In the presence of superoxide radical and hydrogen peroxide, stored ferric iron $\left(\mathrm{Fe}^{3+}\right)$ is reduced to ferrous iron $\left(\mathrm{Fe}^{2+}\right)$, which catalyzes the formation of the hydroxyl radical, which in turn can promote lipid peroxidation, mutagenesis, DNA damage, oncogene activation, and tumor suppressor inhibition, possibly increasing the risk of some cancer (49). Thus, excess iron may result in increasing the risk of cancer (50). The carcinogenicity of iron has been demonstrated in animal models, and epidemiologic studies have shown associations with several cancers (49). For example, pathological conditions such as hemochromatosis, chronic viral hepatitis B and C, exposure to asbestos fibers, as well as endometriosis have been recognized as iron overload-associated risks for cancer (51).

Several studies suggest that oxidative stress is a component of the inflammatory reaction associated with endometriosis (52). Retrograde menstruation and ovarian hemorrhage carry pro-oxidant factors, such as heme and iron (52). Excess iron accumulation can result in toxicity and may be one of the factors contributing to the development of endometriosis and subsequently EAOC (53-55).

There is a genomic site vulnerable to the oxidative damage (56). There are target genes in iron-induced carcinogenesis and that iron-catalyzed oxidative DNA damage is not random (51). In an animal model, p15 (CDKN2B, p15INK4b) and p16 (CDKN2A, p16INK4a) tumor suppressor genes are among the major target genes. Loss of gene architecture at the p15 occurs frequently in malignant cell lines and is common in squamous cell carcinoma of the head and neck (56). Allelic loss of p16 is a genetic change involved in the pathogenesis of smoking-induced human lung cancer and rat renal carcinogenesis.

\section{Conclusions}

A histologically normal ectopic endometrium bears genetic instability or damages caused by iron-dependent oxidative stress. DNA damage and LOH caused by oxidative stress is a critical factor in the carcinogenic process. Genetic instabilitymediated down-regulation of some tumor suppressor genes and overexpression of specific candidate oncogenes are implicated in CCC tumorigenesis. PLK rapidly activated by oxidative stress could induce Emi1 phosphorylation and activation to ensure mitosis entry. Up-regulation of Emi1, K-RAS and mTOR expression is a late event in the development of atypical endometriosis-CCC sequence. $\mathrm{LOH}$ and mutations in the candidate tumor suppressor genes described in Fig. 1 tend to occur frequently in endometriosis-associated CCC. LOH studies have implicated the involvement of many chromosomal regions including 1q, 5q, 9p, 10q, 11q, 17q and 22q. These loci may contain candidate tumor suppressor genes involved in the initiation, promotion and progression of CCC. $\mathrm{LOH}$ in the p53 gene is considered to be a late event in the development of endometriosis-CCC sequence. In the case of CCAF-CCC sequence, the PTEN and APC gene alterations is an early event in tumorigenesis, whereas LOH on $1 p$ and $13 q$ may be involved later in this sequence. Specific tumor suppressor genes that could directly contribute to CCC carcinogenesis have yet to be elucidated.

Patients with CCC have poor prognosis and short survival due to lack of effective therapy. Up-regulated HNF-1ß, Emi1 and mTOR signaling and mutation of K-RAS are found in CCC. An enormous amount of knowledge has been obtained from recent preclinical experiments. Therefore, inhibitors that target HNF-1ß, Emi1, K-RAS and its downstream targets, including RAF, MEK, mTOR and VEGF, for example, could be taken into consideration in patients with CCC. Furthermore, treatment with an iron chelator may be beneficial in endometriosis patients to prevent iron overload, thereby diminishing the development of CCC. Future research is expected to identify new compounds to block important signaling pathways and to identify new targets for therapies through novel high-throughput technologies. The challenge to transferral of these new compounds from preclinical research to disease management is the design of effective prospective randomized clinical trials. Further study is therefore needed to better understand the pathogenesis of and basic therapies for CCC.

\section{References}

1. Christie M and Oehler MK: Molecular pathology of epithelial ovarian cancer. J Br Menopause Soc 12: 57-63, 2006.

2. Prowse AH, Manek S, Varma R, et al: Molecular genetic evidence that endometriosis is a precursor of ovarian cancer. Int J Cancer 119: 556-562, 2006.

3. Kobayashi H, Sumimoto K, Moniwa N, et al: Risk of developing ovarian cancer among women with ovarian endometrioma: a cohort study in Shizuoka, Japan. Int J Gynecol Cancer 17: 37-43, 2007.

4. Yamamoto S, Tsuda H, Takano M, Hase K, Tamai S and Matsubara O: Clear-cell adenofibroma can be a clonal precursor for clear-cell adenocarcinoma of the ovary: a possible alternative ovarian clear-cell carcinogenic pathway. J Pathol 216: 103-110, 2008.

5. Dinulescu DM, Ince TA, Quade BJ, Shafer SA, Crowley D and Jacks T: Role of K-ras and Pten in the development of mouse models of endometriosis and endometrioid ovarian cancer. Nat Med 11: 63-70, 2005. 
6. Amemiya S, Sekizawa A, Otsuka J, Tachikawa T, Saito H and Okai T: Malignant transformation of endometriosis and genetic alterations of K-ras and microsatellite instability. Int J Gynaecol Obstet 86: 371-376, 2004.

7. Tan DS and Kaye S: Ovarian clear cell adenocarcinoma: a continuing enigma. J Clin Pathol 60: 355-360, 2007.

8. Zorn KK, Bonome T, Gangi L, et al: Gene expression profiles of serous, endometrioid, and clear cell subtypes of ovarian and endometrial cancer. Clin Cancer Res 11: 6422-6430, 2005.

9. Jiang X, Hitchcock A, Bryan EJ, Watson RH, Englefield P, Thomas EJ and Campbell IG: Microsatellite analysis of endometriosis reveals loss of heterozygosity at candidate ovarian tumor suppressor gene loci. Cancer Res 56: 3534-3539, 1996.

10. Bischoff FZ, Heard M and Simpson JL: Somatic DNA alterations in endometriosis: high frequency of chromosome 17 and p53 loss in late-stage endometriosis. J Reprod Immunol 55: 49-64, 2002.

11. Goumenou AG, Arvanitis DA, Matalliotakis IM, Koumantakis EE and Spandidos DA: Microsatellite DNA assays reveal an allelic imbalance in p16(Ink4), GALT, p53, and APOA2 loci in patients with endometriosis. Fertil Steril 75: 160-165, 2001

12. Obata $\mathrm{K}$ and Hoshiai $\mathrm{H}$ : Common genetic changes between endometriosis and ovarian cancer. Gynecol Obstet Invest 50 (Suppl. 1): S39-S43, 2000.

13. Thomas EJ and Campbell IG: Molecular genetic defects in endometriosis. Gynecol Obstet Invest 50 (Suppl. 1): S44-S50, 2000

14. Varma R, Rollason T, Gupta JK and Maher ER: Endometriosis and the neoplastic process. Reproduction 127: 293-304, 2004.

15. Viganó P, Somigliana E, Chiodo I, Abbiati A and Vercellini P. Molecular mechanisms and biological plausibility underlying the malignant transformation of endometriosis: a critical analysis. Hum Reprod Update 12: 77-89, 2006.

16. Sato N, Tsunoda H, Nishida M, Morishita Y, Takimoto Y, Kubo T and Noguchi M: Loss of heterozygosity on 10q23.3 and mutation of the tumor suppressor gene PTEN in benign endometrial cyst of the ovary: possible sequence progression from benign endometrial cyst to endometrioid carcinoma and clear cell carcinoma of the ovary. Cancer Res 60: 7052-7056, 2000.

17. Martini M, Ciccarone M, Garganese G, et al: Possible involvement of hMLH1, p16(INK4a) and PTEN in the malignant transformation of endometriosis. Int J Cancer 102: 398-406, 2002.

18. Cody NA, Ouellet V, Manderson EN, et al: Transfer of chromosome 3 fragments suppresses tumorigenicity of an ovarian cancer cell line monoallelic for chromosome $3 \mathrm{p}$ Oncogene 26: 618-632, 2007.

19. Miller YE, Minna JD and Gazdar AF: Lack of expression of aminoacylase-1 in small cell lung cancer. Evidence for inactivation of genes encoded by chromosome $3 \mathrm{p}$. J Clin Invest 83: 2120-2124, 1989.

20. Angeloni D: Molecular analysis of deletions in human chromosome 3 p 21 and the role of resident cancer genes in disease. Brief Funct Genomic Proteomic 6: 19-39, 2007.

21. Serrano M, Hannon GJ and Beach D: A new regulatory motif in cell-cycle control causing specific inhibition of cyclin D/CDK4. Nature 366: 704-707, 1993.

22. Schuyer M, van Staveren IL, Klijn JG, et al: Sporadic CDKN2 (MTS1/p16ink4) gene alterations in human ovarian tumours. Br J Cancer 74: 1069-1073, 1996.

23. O'Neill CJ and McCluggage WG: p16 expression in the female genital tract and its value in diagnosis. Adv Anat Pathol 13: 8-15, 2006.

24. Shimizu M, Nikaido T, Toki T, Shiozawa T and Fujii S: Clear cell carcinoma has an expression pattern of cell cycle regulatory molecules that is unique among ovarian adenocarcinomas. Cancer 85: 669-677, 1999.

25. Ali-Fehmi R, Khalifeh I, Bandyopadhyay S, et al: Patterns of loss of heterozygosity at 10q23.3 and microsatellite instability in endometriosis, atypical endometriosis, and ovarian carcinoma arising in association with endometriosis. Int J Gynecol Pathol 25: 223-229, 2006

26. Obata K, Morland SJ, Watson RH, Hitchcock A, ChenevixTrench G, Thomas EJ and Campbell IG: Frequent PTEN/ MMAC1 mutations in endometrioid but not serous or mucinous epithelial ovarian tumors. Cancer Res 58: 2095-2097, 1998.

27. Willner J, Wurz K, Allison KH, Galic V, Garcia RL, Goff BA and Swisher EM: Alternate molecular genetic pathways in ovarian carcinomas of common histological types. Hum Pathol 38: 607-613, 2007
28. Martin ES, Cesari R, Pentimalli F, et al: The BCSC-1 locus at chromosome 11q23-q24 is a candidate tumor suppressor gene. Proc Natl Acad Sci USA 100: 11517-11522, 2003.

29. Melin A, Sparén P, Persson I and Bergqvist A: Endometriosis and the risk of cancer with special emphasis on ovarian cancer. Hum Reprod 21: 1237-1242, 2006.

30. Ramos D and Aldaz CM: WWOX, a chromosomal fragile site gene and its role in cancer. Adv Exp Med Biol 587: 149-159, 2006.

31. Sáinz de la Cuesta R, Izquierdo M, Cañamero M, Granizo JJ and Manzarbeitia F: Increased prevalence of p53 overexpression from typical endometriosis to atypical endometriosis and ovarian cancer associated with endometriosis. Eur J Obstet Gynecol Reprod Biol 113: 87-93, 2004.

32. Akahane T, Sekizawa A, Purwosunu Y, Nagatsuka M and Okai T: The role of p53 mutation in the carcinomas arising from endometriosis. Int J Gynecol Pathol 26: 345-351, 2007.

33. Dion F, Mes-Masson AM, Seymour RJ, Provencher D and Tonin PN: Allelotyping defines minimal imbalance at chromosomal region $17 \mathrm{q} 25$ in non-serous epithelial ovarian cancers. Oncogene 19: 1466-1472, 2000

34. Gogusev J, Bouquet de Jolinière J, Telvi L, Doussau M, Stojkoski A and Levardon M: Cellular and genetic constitution of human endometriosis tissues. J Soc Gynecol Investig 7: 79-87, 2000.

35. Pal T, Permuth-Wey J, Kumar A and Sellers TA: Systematic review and meta-analysis of ovarian cancers: estimation of microsatellite-high frequency and characterization of mismatch repair deficient tumor histology. Clin Cancer Res 14: 6847-6854, 2008.

36. Chang CL, Marra G, Chauhan DP, et al: Oxidative stress inactivates the human DNA mismatch repair system. Am J Physiol Cell Physiol 283: C148-C154, 2002.

37. Sekizawa A, Amemiya S, Otsuka J, Saito H, Farina A, Okai T and Tachikawa T: Malignant transformation of endometriosis: application of laser microdissection for analysis of genetic alterations according to pathological changes. Med Electron Microsc 37: 97-100, 2004.

38. Kato N, Sasou S and Motoyama T: Expression of hepatocyte nuclear factor-1beta (HNF-1beta) in clear cell tumors and endometriosis of the ovary. Mod Pathol 19: 83-89, 2006.

39. Köbel M, Kalloger SE, Carrick J, et al: A limited panel of immunomarkers can reliably distinguish between clear cell and high-grade serous carcinoma of the ovary. Am J Surg Pathol 33: 14-21, 2009.

40. Kato N, Tamura $\mathrm{G}$ and Motoyama T: Hypomethylation of hepatocyte nuclear factor-1beta (HNF-1beta) $\mathrm{CpG}$ island in clear cell carcinoma of the ovary. Virchows Arch 452: 175-180, 2008.

41. Tsuchiya A, Sakamoto M, Yasuda J, et al: Expression profiling in ovarian clear cell carcinoma: identification of hepatocyte nuclear factor- 1 beta as a molecular marker and a possible molecular target for therapy of ovarian clear cell carcinoma. Am J Pathol 163: 2503-2512, 2003.

42. Senkel S, Lucas B, Klein-Hitpass L and Ryffel GU: Identification of target genes of the transcription factor HNF1beta and HNF1alpha in a human embryonic kidney cell line. Biochim Biophys Acta 1731: 179-190, 2005

43. Tang L, Wang TT, Wu YT, Zhou CY and Huang HF: High expression levels of cyclin B1 and Polo-like kinase 1 in ectopic endometrial cells associated with abnormal cell cycle regulation of endometriosis. Fertil Steril (In press).

44. Hsu JY, Reimann JD, Sorensen CS, et al: E2Fdependent accumulation of hEmi1 regulates $S$ phase entry by inhibiting APC(Cdh1). Nat Cell Biol 4: 358-366, 2002.

45. Gütgemann I, Lehman NL, Jackson PK and Longacre TA: Emi1 protein accumulation implicates misregulation of the anaphase promoting complex/cyclosome pathway in ovarian clear cell carcinoma. Mod Pathol 21: 445-454, 2008.

46. Yagyu T, Tsuji Y, Haruta S, et al: Activation of mammalian target of rapamycin in postmenopausal ovarian endometriosis. Int J Gynecol Cancer 16: 1545-1551, 2006.

47. Jin T, George Fantus I and Sun J: Wnt and beyond Wnt: multiple mechanisms control the transcriptional property of beta-catenin. Cell Signal 20: 1697-1704, 2008.

48. Valko M, Izakovic M, Mazur M, Rhodes CJ and Telser J: Role of oxygen radicals in DNA damage and cancer incidence. Mol Cell Biochem 266: 37-56, 2004.

49. Kabat GC and Rohan TE: Does excess iron play a role in breast carcinogenesis? An unresolved hypothesis. Cancer Causes Control 18: 1047-1053, 2007. 
50. Huang X: Iron overload and its association with cancer risk in humans: evidence for iron as a carcinogenic metal. Mutat Res 533: 153-171, 2003.

51. Toyokuni S: Role of iron in carcinogenesis: Cancer as a ferrotoxic disease. Cancer Sci (In press).

52. Van Langendonckt A, Casanas-Roux F and Donnez J: Oxidative stress and peritoneal endometriosis. Fertil Steril 77: 861-870, 2002.

53. Defrère S, Lousse JC, González-Ramos R, Colette $\mathrm{S}$, Donnez $\mathrm{J}$ and van Langendonckt A: Potential involvement of iron in the pathogenesis of peritoneal endometriosis. Mol Hum Reprod 14: 377-385, 2008.
54. Kobayashi H, Yamada Y, Kanayama S, et al: The role of iron in the pathogenesis of endometriosis. Gynecol Endocrinol (In press). 55. Yamaguchi K, Mandai M, Toyokuni S, Hamanishi J, Higuchi T, Takakura K, Fujii S: Contents of endometriotic cysts, especially the high concentration of free iron, are a possible cause of carcinogenesis in the cysts through the iron-induced persistent oxidative stress. Clin Cancer Res 14: 32-40, 2008.

56. Hiroyasu M, Ozeki M, Kohda H, Echizenya M, Tanaka T, Hiai H and Toyokuni S: Specific allelic loss of p16 (INK4A) tumor suppressor gene after weeks of iron-mediated oxidative damage during rat renal carcinogenesis. Am J Pathol 160: 419-424, 2002. 